\title{
Changes in distributions of waist circumference, waist-to-hip ratio and waist- to-height ratio over an 18-year period among Chinese adults: a longitudinal study using quantile regression
}

\author{
Xiwen Qian', Chang Su², Bing Zhang ${ }^{2}$, Guoyou Qin', Huijun Wang ${ }^{2 *}$ and Zhenyu Wu ${ }^{1 *}$ (i)
}

\begin{abstract}
Background: Little is known about the long-term shifts in distributions of three abdominal-obesity-related indicators, waist circumference (WC), waist-to-hip ratio (WHpR) and waist-to-height ratio (WHtR) among Chinese adults. Traditional mean regression models used in the previous analyses were limited in their ability to capture cross-distribution among effects. The current study aims to describe the shift in distribution of WC, WHpR, and WHtR over a period of 18 years (1993-2011) in China, and to reveal quantile-specific associations of the three indicators with key covariates.
\end{abstract}

Methods: Longitudinal data from seven waves of the China Health and Nutrition Surveys (CHNS) in 1993, 1997, 2000, 2004, 2006, 2009 and 2011 were analyzed. The LMS method was used to illustrate the gender-specific quantile curves of WC, WHtR and WHpR over age. Separate gender-stratified longitudinal quantile regressions were employed to investigate the effect of important factors on the trends of the three indicators.

Results: A total of 11,923 participants aged 18-65 years with 49,507 observations were included in the analysis. The density curves of WC, WHtR and WHpR shifted to right and became wider. The three outcomes all increased with age and increased more at upper percentiles. From the multivariate quantile regression, physical activity was negatively associated in both genders; smoking only had a negative effect on male indicators. Education and drinking behavior both had opposite effects on the three indicators between men and women. Marital status and income were positively associated with the shifts in WC, WHtR and WHpR in male and female WC, while urbanicity index had a positive effect on three outcomes in men but inconsistent effect among female outcomes.

Conclusions: The abdominal-obesity related indicators of the Chinese adults experienced rapid growth according to our population-based, age- and gender-specific analyses. Over the 18-year study period, major increases in WC, WHtR and WHpR were observed among Chinese adults. Specifically, these increases were greater at upper percentiles and in men. Age, physical activity, energy intake, drinking, smoking, education, income and urbanicity index were associated with elevated abdominal obesity indicators, and the effects differed among percentiles and between genders.

Keywords: Waist circumference, Waist-to-hip ratio, Waist-to-height ratio, Abdominal obesity, Quantile regression

\footnotetext{
*Correspondence: wanghj@ninh.chinacdc.cn; zyw@fudan.edu.cn

${ }^{2}$ National Institute for Nutrition and Health, Chinese Center for Disease Control and Prevention, 29 Nanwei Road, Xicheng District, Beijing 100050, People's Republic of China

'Department of Biostatistics, School of Public Health, Key Laboratory of Public Health Safety and Collaborative Innovation Center of Social Risks Governance in Health, Fudan University, Shanghai 200032, People's Republic of China
}

(c) The Author(s). 2019 Open Access This article is distributed under the terms of the Creative Commons Attribution 4.0 International License (http://creativecommons.org/licenses/by/4.0/), which permits unrestricted use, distribution, and reproduction in any medium, provided you give appropriate credit to the original author(s) and the source, provide a link to the Creative Commons license, and indicate if changes were made. The Creative Commons Public Domain Dedication waiver (http://creativecommons.org/publicdomain/zero/1.0/) applies to the data made available in this article, unless otherwise stated. 


\section{Background}

Obesity has become a serious problem that threatens public health. Body mass index (BMI), which is defined as weight divided by the square of the height $\left(\mathrm{kg} / \mathrm{m}^{2}\right)$, is probably the most commonly used index to evaluate overall body fatness and to determine overweight or obesity in adults. However, one major drawback of BMI is that it fails to consider the distribution of fat throughout the body. Abdominal fat, i.e., that around the heart, liver and kidneys, has been found by no means to be less pathogenic than general obesity, but rather has more significant relationship with heavy disease burden [1-3].

Abdominal obesity can usually be evaluated by the three most popularly used indicators: waist circumference (WC), waist-to-hip ratio (WHpR) and waist-to-height ratio (WHtR). Significant increases in these indicators have been reported in developed countries. An Australian 12-year cohort study reported increases of $4.32 \mathrm{~cm}$ and $6.25 \mathrm{~cm}$ in WC from 1999 to 2011 for men and women, respectively [4]. The prevalence of WC over $102 \mathrm{~cm}$ among men aged 40-79 years has increased by $13.1 \%$ in northeast European cities from 2003 to 2010 [5]. In the US, the overall age adjusted mean WC increased $3 \mathrm{~cm}$ from 1999 to 2012 [6]. Developing countries such as China have also experienced a serious obesity crisis. The prevalence of abdominal obesity thus increased dramatically from 17.3 to $39.4 \%$ between 1997 and 2009 [3]. The age-adjusted prevalence of abdominal obesity in China was $35.3 \%$ in men and $51.7 \%$ in women in 2011 [7].

More importantly, accumulating evidence has shown the positive correlation of abdominal obesity indicators with the risk of chronic diseases. For example, WC alone or WC combined with BMI is more predictive than BMI alone for hypertension [8] and obesity-related mortality [9]. WHtR is a better predictor of metabolic syndrome [10], diabetes, hypertension and cardiovascular disease (CVD) $[11,12]$. WHpR is thought to more precisely measure visceral fat because it attenuates the influence of subcutaneous fat by considering hip circumference (HC) $[13,14]$, which is also inversely connected to dyslipidemia, diabetes and CVD [15]. Although the optimal predictive abdominal obesity indicators for chronic diseases varied among studies, what remains certain is that these three indicators are negatively associated with various diseases.

The past decades witnessed great development in China. The popularizing of mass education and the process of urbanization had a dramatic influence on people's health. The health status of Chinese population was challenged by changes in dietary pattern and lifestyle. Studies also reported the important association between socioeconomic status and obesity [16, 17]. Therefore, socioeconomic and lifestyle factors were considered in our study, to further explore the association of these variables and changes of abdominal obesity related measures.

Traditionally, a general linear regression model was frequently performed to study the effect of obesity-related covariates on the conditional mean of the dependent variable. However, this method is not suitable if the effect of explanatory variables differs at different levels of the outcome, and cannot make full use of the overall distribution. By contrast, quantile regression builds an array of equations that are regressed to defined quantiles without extra hypotheses in distribution. Thus, it is more robust against outliers or skewness to the response variable than is ordinary linear regression [18]. Meanwhile, quantile regression can provide a detailed description of the association between covariates and each quantile of the response variable.

In the current study, we aim to describe the secular shift of abdominal obesity in adults, as depicted by WC, WHtR and WHpR, and to explore the relationships between covariates and changes of indicators at each quantile. Our results provide new perspectives on the population health and may encourage researchers and policy makers to control, prevent and decrease the epidemic of abdominal obesity.

\section{Methods}

\section{Study population}

Data for this study was derived from the China Health and Nutrition Surveys (CHNS), a large-scale longitudinal survey designed to cover key public health risk factors and health outcomes and, demographic, socioeconomic factors at the individual, household and community levels. The CHNS aimed to examine the effects of social and economic change across time on public health. The sample of this large-scale survey was selected randomly from eight provinces in the first wave in 1989. Within each province, stratified sampling was used to select cities and counties. In later survey years, more provinces were involved, and more data was collected. Detailed information is available in the profile $[19,20]$.

In the current study, adults aged 18 to 65 from the seven latest waves in 1993, 1997, 2000, 2004, 2006, 2009 and 2011 were analyzed. Due to the longitudinal nature of our study, only subjects with more than one records were considered as qualified participants. We excluded subjects without measurements of WC, height or $\mathrm{HC}$ and participants whose WC was outside the range of 45 to $150 \mathrm{~cm}$, whose $\mathrm{HC}$ was outside the range of 55 to $150 \mathrm{~cm}$, or whose height was outside the range of 120 to $200 \mathrm{~cm}$ were also excluded as extreme outliers. Missing values were imputed with multiple imputation method. A total of 11,923 individuals with 49,507 records were involved in our final analysis. 


\section{Outcomes}

The outcomes of interest were WC, WHtR and WHpR. $\mathrm{WC}, \mathrm{HC}$ and height were collected by physical measurements methods. WC was taken at a midpoint between the bottom of the rib cage and the top of the iliac crest at the end of exhalation. $\mathrm{HC}$ was taken at the level of maximal gluteal protrusion. Both $\mathrm{WC}$ and $\mathrm{HC}$ were measured using a SECA tape to the nearest $0.1 \mathrm{~cm}$. Height was measured without shoes to the nearest 0.2 $\mathrm{cm}$ using a portable stadiometer. WHtR and WHpR were computed as WC divided by height and $\mathrm{HC}$, respectively.

\section{Covariates}

Categorical covariates included sex (male, female), educational level (none or primary school, middle school, senior school or above), smoking status (no, yes), drinking status (no, yes) and marital status (unmarried, married, divorced and other). Continuous covariates included age, energy intake, total physical activity, per capita family annual income, and urbanicity level.

Data of smoking, drinking, energy intake, physical activity, educational level, marital status and income was collected by questionnaires and dietary survey. Variables were defined as follows:

Energy intake average daily energy intake for each individual, calculated based on the data of detailed food consumption during three consecutive days at both the household and individual level.

Physical activities (PA) indicated by average metabolic equivalents of task (MET) hours per day in a week, estimated from four aspects: occupational, domestic, active leisure and travel. The MET is defined as the ratio of a person's working metabolic rate (and therefore the rate of energy consumption) to his or her basal metabolic rate. One MET is defined as $1 \mathrm{kcal} / \mathrm{kg}$-hour of energy cost.

Per capita income average individual income, calculated based on reported gross annual household income and was inflated to 2011 values using the Consumer Price Index [21] and categorized into year-specific tertiles.

Urbanicity index A total score at the community level to describe the characteristics and degree of urbanization, calculated by a multicomponent continuous scale developed specifically for the CHNS [22]. Each community was evaluated by 12 components with a maximum of 10 points of each, including economic activity, traditional markets, modern markets, population density, transportation infrastructure, communications, sanitation, health infrastructure, housing, education, diversity and social services. This variable was categorized into tertiles in the regression models.

\section{Statistical analysis}

The demographic, socioeconomic and lifestyle features in each wave were described in Table 1 . Continuous variables were expressed as medians, the first quartile (25th) and the third quartile (75th). Categorical variables were presented with frequency and percentage. We used trend Chi-square test for categorical variables and Kruskal-Wallis test for continuous variables to examine the difference over time.

To illustrate the shifts of WC, WHtR and WHpR between 1993 and 2011, kernel density plots were used to display distributions. To illustrate the age-specific smoothed quantile curves for these three indicators, LMS (lambda, mu, and sigma) quantile regression were constructed, in which the parameters $\lambda, \mu$ and $\sigma$ were chosen to maximize a penalized log-likelihood in the VGAM package in $\mathrm{R}$ version 3.3.2 ( $\mathrm{R}$ Development Core Team, Vienna, Austria).

Finally, gender-stratified longitudinal analyses were conducted to investigate the time trend of abdominal obesity measures and influencing factors. Due to limited space, we multiplied both WHtR and WHpR by 100 to avoid the regression coefficients close to zero. Longitudinal quantile regression models with fixed effects for each outcome of interest were built in three steps using the lqmm package: Model 1 only included year, and the coefficient measured the crude yearly change of the outcome; Model 2 adjusted individual-level features, including age, energy intake, PA, smoking status and drinking status, educational level, marital status and per capita annual income, so the coefficient reflected the yearly change conditional on individual-level covariates; Model 3 controlled a community-level urbanicity index based on Model 2. Thus, in Model 3, the coefficient of time indicated the effect of time-varying factors or unavailable or unmeasurable covariates like culture, environment and social policy, after controlling individual level and community level covariates. A quadratic term of age was included in Model 2 and Model 3.

\section{Results}

The individual-, household-, and community-level characteristics of the studied samples are presented in Table 1. Approximately one-third of the participants reported smoking or drinking history in each round of the survey. The daily energy intake and total PA showed a decreased trend. WC, the fundamental predictor of abdominal obesity, increased by almost $10 \mathrm{~cm}$, whereas the median $\mathrm{HC}$ and height increased $6 \mathrm{~cm}$ and $1.6 \mathrm{~cm}$, respectively, over an 18-year period. 


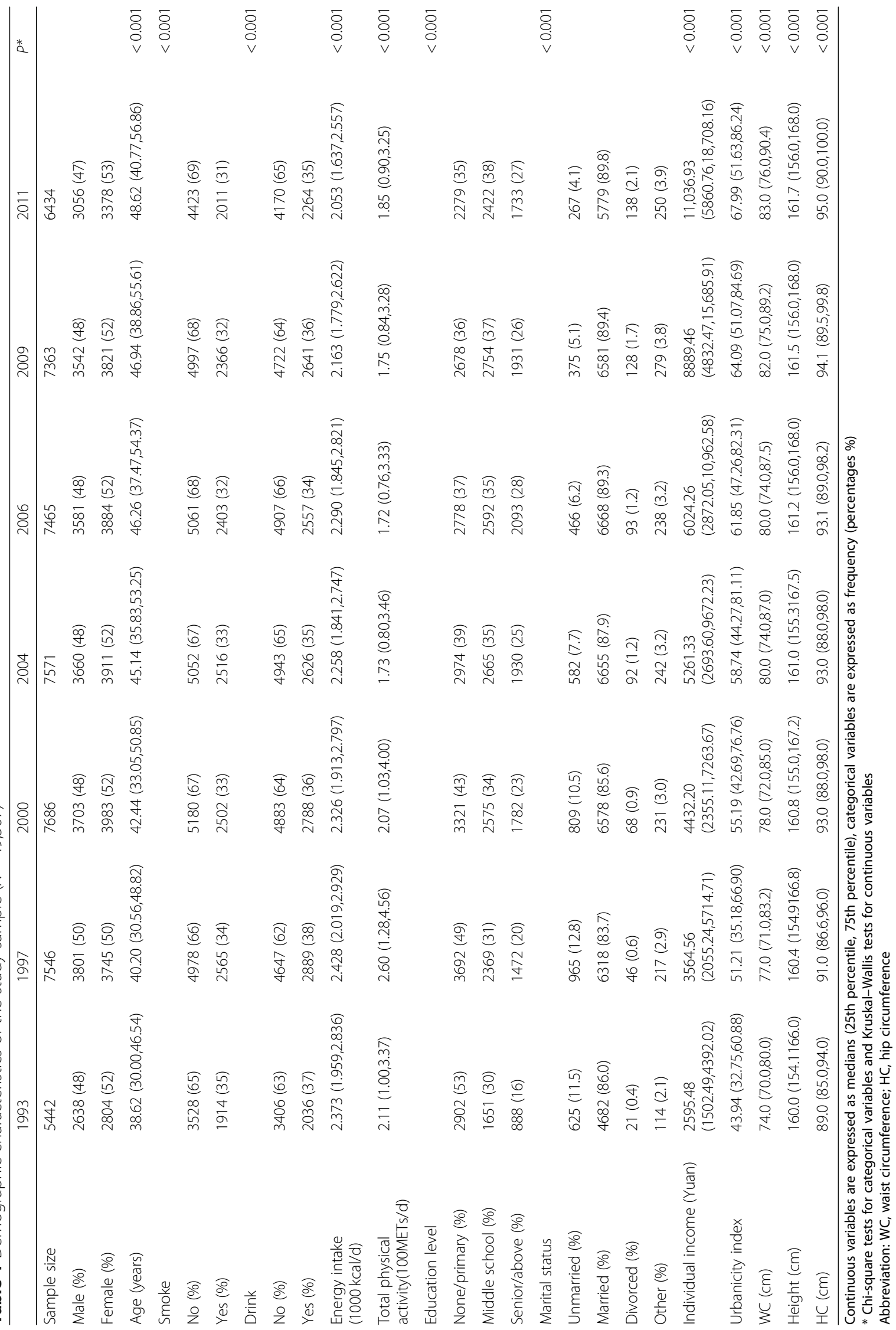




\section{Trends of the distribution changes in WC, WHtR and} WHpR from 1993 to 2011

For both genders, from 1993 to 2011, the density curves all shifted to right and became wider, which meant that the proportion of subjects with high WC, WHtR and WHpR increased with time (Fig. 1; in Additional file 1: Figure S1). However, greater increases in WC, WHtR and WHpR were found in men than in women.

\section{Shifts in the quartile curves of WC, WHtR and WHpR}

Percentile curves, using the LMS method, for both genders in each age group were shown in selected years (Fig. 2; in Additional file 1: Figure S1- S4). All the solid lines (2011) were above the dotted lines (1993) (Fig. 2), which is in consistence with the results in Fig. 1 showing that people were getting fatter in 2011. Specifically, for the quartile levels of WC, WHtR increased dramatically with age, while WHpR was relatively stable. Note that WC, WHtR and WHpR increased almost linearly in women. In men, the curves were not as smooth and became flat or even decreased with age after 50 years of age.

\section{Gender-specific quantile regression results for each outcome}

Time effects, which were estimated by the yearly coefficients in three quantile models using WC, WHtR or WHpR as an outcome, are listed in Table 2. Model 1 suggested a significant increase in all the outcomes of interest from 10th percentile to the 90th percentile for both sexes. WC increased more at upper percentiles while WHpR increased more at lower percentiles. For example, WC increased $1.400 \mathrm{~cm}$ (95\% CI: 1.286, 1.514) and $1.227 \mathrm{~cm}(95 \% \mathrm{CI}: 1.091,1.360)$ per year at 10th percentile in men and women, respectively; at 90th percentile, WC increased $1.507 \mathrm{~cm}$ (95\% CI: $1.327,1.686)$ and $1.337 \mathrm{~cm}$ (95\% CI: $1.209,1.460)$ in men and women. After adjusting for lifestyle and socioeconomic variables in Model 2, the time effect on the three outcomes declined but still remained significant (Table 2). When the urbanicity index was considered in Model 3, the time effect on male WHtR, WHpR and female WC became slightly stronger compared with Model 2, while on other declined at all percentiles.
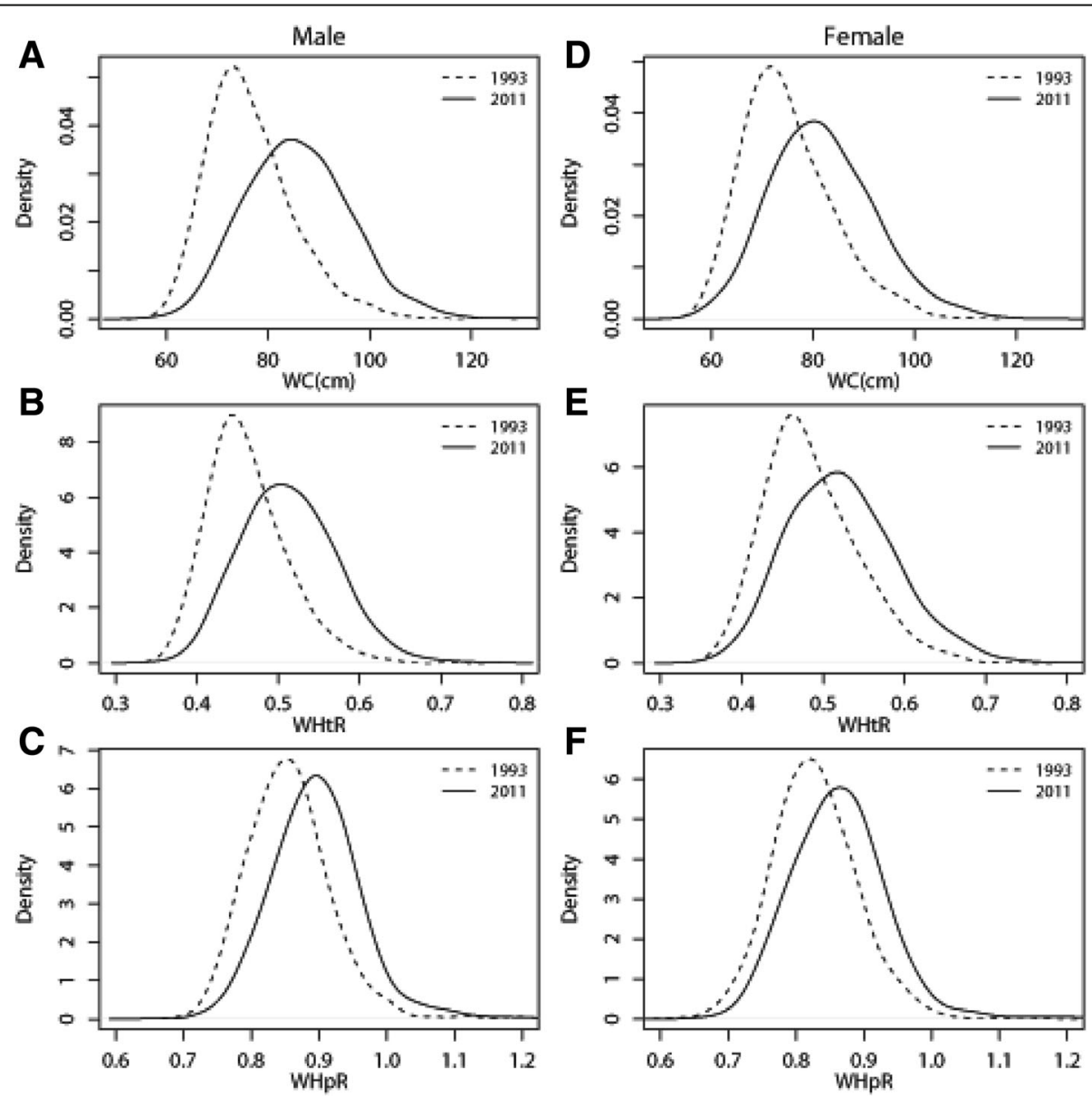

Fig. 1 Shifts in distributions of WC, WHtR and WHpR for Chinese adults between 1993 and 2011 
Detailed information of Model 3 is provided in (Additional file 1 :Table S1-S3). Among the three outcomes that all increased with age and increased more at upper percentiles, the increases in WC, male WHtR and male WHpR were at a decreasing speed as the coefficients of quadratic age were significantly negative. Results show that the estimates from least squared model are different from quantile regression.

Physical activities had a significant negative association -- which was much stronger at lower percentiles than that at upper percentiles -- with WC, WHtR and WHpR in both men and women. For example, female WC decreased $0.305 \mathrm{~cm}$ (95\% CI: -0.385 , $0.225)$ and $0.236 \mathrm{~cm}(95 \% \mathrm{CI}:-0.335,-0.137)$ with an additional 100 MET-hour of physical activity per day at 10th and 90th percentiles, respectively; female 100-fold WHtR and WHpR decreased 0.145 (95\% CI: $-0.187,-0.103)$ and 0.167 (95\% CI: -0.217, - 0.115) respectively at 10 th percentile, and 0.105 (95\% CI: $-0.151,-0.059)$ and 0.124 (95\% CI: $-0.183,-0.066)$ respectively at 90th percentile, with an additional 10,000 MET-hour of physical activity per day. Smoking only significantly associated with male WC and WHtR. WC in male smokers tended to be $1.061 \mathrm{~cm}$ (95\% CI: $-1.470,-0.652$ ) smaller than that in nonsmokers at 10th percentile, and $1.044 \mathrm{~cm}(95 \% \mathrm{CI}$ : $-1.453,-0.635)$ at 90th percentile. WHtR in male smokers was also smaller, and the negative effect of smoking was stronger at lower percentiles.

Interestingly, drinking and educational level had completely opposite effects on the three outcomes between sexes. Drinking caused a significant increase in all percentiles of WC, WHtR and WHpR among men. While female non-drinkers with media value of WC were prone to have $0.599 \mathrm{~cm}(95 \% \mathrm{CI}$ : -1.091, - 0.107) larger WC, as well as larger WHtR and WHpR, but significance was only shown in WC. Higher educational level tended to cause larger WC and WHtR in men, but smaller WC, WHtR and WHpR in women. The effects of education at upper percentiles were stronger than at lower percentiles in men, but weaker in women. For instance, men receiving senior schooling or above had approximately $1.775 \mathrm{~cm}(95 \%$ CI: $1.221,2.329)$ more of WC at 10th percentiles and $1.790 \mathrm{~cm}(95 \%$ CI: 1.235, 2.344) at 90th percentile, compared with those with no or primary schooling. For women with senior schooling or above, WC at 10th percentile was $2.257 \mathrm{~cm}(95 \% \mathrm{CI}:-2.816,-1.698)$ and at 90th percentile was $2.250 \mathrm{~cm}(95 \% \mathrm{CI}:-2.809,-1.690)$ smaller than those least-educated.

Compared with never-married men and women, the married had notably larger outcomes, and the effects of marriage on three indicators were stronger in men than in women. WC in married men and women were around $2.116 \mathrm{~cm}$ (95\% CI at 50th percentile: 1.452, 2.780) and
$1.651 \mathrm{~cm} \mathrm{(95 \%} \mathrm{CI} \mathrm{at} \mathrm{50th} \mathrm{percentile:} 0.959,2.342)$, respectively larger than that in unmarried men and women. Higher level of income was positively associated with three indicators in men, but the association was inconsistent among three outcomes in women. For example, men with relatively high income had $1.913 \mathrm{~cm}$ (95\% CI: 1.561, 2.266) lager WC at 10th percentiles and $1.926 \mathrm{~cm}(95 \% \mathrm{CI}: 1.574,2.278)$ at 90 th percentile. In women, high level of income contributed to an increase by $0.603 \mathrm{~cm}(95 \% \mathrm{CI}$ at 50 th percentile: $0.243,0.963)$ in WC but a decrease in WHpR. For men, urbanicity index had a positive effect which was stronger at upper percentiles on the WC, WHtR and WHpR. Living in a community with high urbanicity index resulted in an increment of $1.651 \mathrm{~cm}(95 \%$ CI: 1.577, 2.743) in WC at 10th percentile in men and $1.667 \mathrm{~cm}$ (95\% CI: 1.127 , $2.207)$ at 90th percentile, compared with men living in a community with low urbanicity index. Similar results were also found in women for WC, but not WHtR and WHpR.

\section{Discussion}

To the best of our knowledge, the current study is the first age- and gender-specific analysis in a Chinese population to explore the secular trends of three indicators of abdominal obesity and their relation with potential risk factors. We found the three indicators increased more in men and at upper percentiles over a period of 18 years.

Previous studies using CHNS data showed that WC and the prevalence of abdominal obesity had increased greatly from 1993 to 2009 in both sexes [23], especially among those living in rural regions and among individuals aged 40-59 years [24]. Similar trends for WC and WHpR were found in US adults $[6,25]$ and the Finnish population [26]. Studies on the change of WHtR and WHpR are limited. We believe that WHtR and WHpR are important for evaluating abdominal obesity. WHtR adjusts for height, which tends to decrease with age and differs among ethnic groups and regions, and WHpR distinguishes "pear-shaped" from "apple-shaped" body types. According to our analyses, WC, WHtR and WHpR have increased rapidly over the past decades, but the patterns of age-specific increase are different. WHtR behaved similarly to WC because there was little change in population height over the decades. The WHpR curves were relatively flat, which means that the WHpR was not as dramatically increased as the other two indicators. The reason for this phenomenon may be that the increase of $\mathrm{WC}$ is usually accompanied by an increase in $\mathrm{HC}$, which makes their ratio more stable. Therefore, WHpR may not be appropriate to describe the secular trend of abdominal obesity, though it is a valuable prognostic indicator for many chronic diseases. 

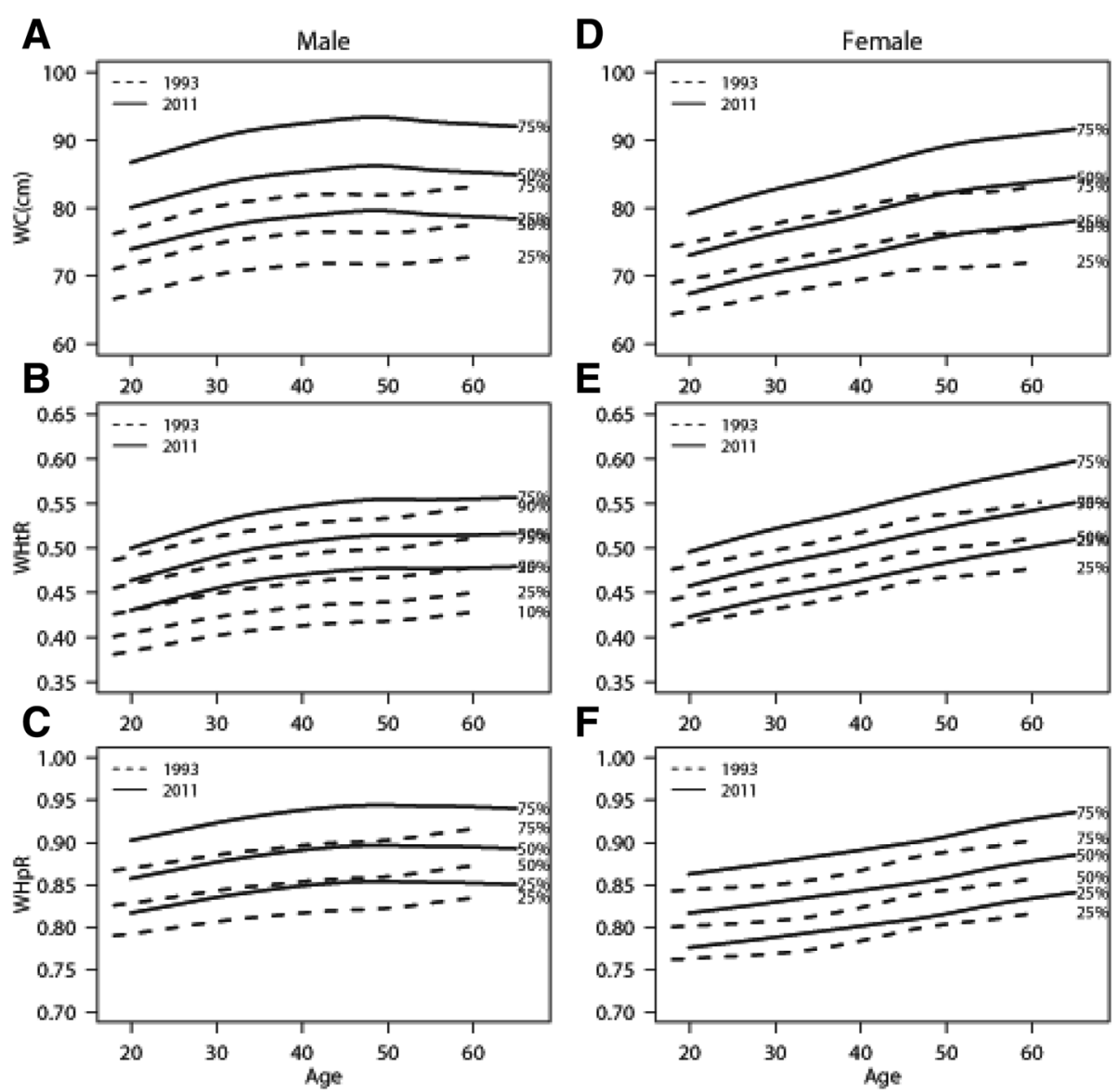

Fig. 2 Quantile curves by age of WC, WHtR and WHpR for adults in 1993 and 2011

Gender-related differences in the shifting patterns of the three indicators can be summarized from two aspects: on the one hand, the extent of increment is greater in men. For example, with WC, the percentile curves in 2011 were much higher than those in 1993 in men, but the extent of the increase in women was not as remarkable as that in men. On the other hand, the age-specific increase is different. Again, taking WC as an example, percentile curves for men started to decrease after 50 years of age, but the curves were still climbing in women. It has been confirmed that gender is an important factor influencing body composition and the accumulation and distribution of body fat due to the effect of sex hormones [27]; it may also be due to different lifestyles.

In the multivariate quantile regression analyses, the opposite effects of education level on the indicators among genders have been noted. Such a divergence has also been reported in other population [4, 28]. A systematic review concluded from numerous related studies that the effect of education on obesity usually depends on the country's level of development and on a modification by gender [17]. It remains possible that in China, women with a high level of education are more likely to live a healthy life because of their knowledge of good health practices [28]; thus, they may have lower abdominal obesity indicators. However, this is different for men, as highly educated men may have busier work schedules, more business trips, more social engagements and business dinners, leading to more energy intake and less time for physical activities. Speaking of income, probably both men and women with high income have busier work thus pay less attention to exercise and healthy diet. Our finding about the association between urbanicity and abdominal obesity indicators was consistent with other studies from China [29] and other developing countries like India [30]. A high level of community urbanicity means good access to food, especially fast food, heavy use of motorized transportation and less open space, which may help explain the increasing abdominal obesity indicators.

The positive association between marital status and obesity or abdominal obesity has been reported [31, 32]. It can be explained by the fact that single people have stronger intention of weight loss to stay attractive while married people tend to have less time for physical 
Table 2 Yearly coefficients (standard error) in quantile regressions using WC, WHtR or WHpR as outcomes

\begin{tabular}{|c|c|c|c|c|c|}
\hline & 10th & 25th & 50th & 75th & 90th \\
\hline \multicolumn{6}{|l|}{ Male } \\
\hline \multicolumn{6}{|l|}{ WC } \\
\hline Model 1 & $1.400^{\mathrm{a}}(0.057)$ & $1.279^{\mathrm{a}}(0.070)$ & $1.337^{\mathrm{a}}(0.035)$ & $1.440^{\mathrm{a}}(0.041)$ & $1.507^{\mathrm{a}}(0.089)$ \\
\hline Model 2 & $1.107^{\mathrm{a}}(0.032)$ & $1.110^{\mathrm{a}}(0.034)$ & $1.133^{\mathrm{a}}(0.032)$ & $1.184^{\mathrm{a}}(0.033)$ & $1.176^{\mathrm{a}}(0.032)$ \\
\hline Model 3 & $1.102^{\mathrm{a}}(0.042)$ & $1.114^{\mathrm{a}}(0.038)$ & $1.152^{\mathrm{a}}(0.039)$ & $1.160^{\mathrm{a}}(0.045)$ & $1.204^{\mathrm{a}}(0.039)$ \\
\hline \multicolumn{6}{|l|}{$\mathrm{WH} t \mathrm{R}$} \\
\hline Model 1 & $0.798^{\mathrm{a}}(0.021)$ & $0.750^{a}(0.024)$ & $0.795^{a}(0.023)$ & $0.800^{a}(0.024)$ & $0.750^{\mathrm{a}}(0.034)$ \\
\hline Model 2 & $0.535^{\mathrm{a}}(0.023)$ & $0.543^{\mathrm{a}}(0.023)$ & $0.552^{\mathrm{a}}(0.022)$ & $0.558^{\mathrm{a}}(0.022)$ & $0.572^{\mathrm{a}}(0.023)$ \\
\hline Model 3 & $0.546^{\mathrm{a}}(0.022)$ & $0.551^{\mathrm{a}}(0.021)$ & $0.560^{\mathrm{a}}(0.020)$ & $0.567^{a}(0.020)$ & $0.579^{\mathrm{a}}(0.021)$ \\
\hline \multicolumn{6}{|l|}{ WHpR } \\
\hline Model 1 & $0.714^{\mathrm{a}}(0.037)$ & $0.671^{\mathrm{a}}(0.022)$ & $0.650^{a}(0.028)$ & $0.664^{\mathrm{a}}(0.022)$ & $0.632^{\mathrm{a}}(0.041)$ \\
\hline Model 2 & $0.461^{\mathrm{a}}(0.027)$ & $0.476^{\mathrm{a}}(0.029)$ & $0.487^{a}(0.028)$ & $0.495^{\mathrm{a}}(0.029)$ & $0.512^{\mathrm{a}}(0.028)$ \\
\hline Model 3 & $0.471^{\mathrm{a}}(0.026)$ & $0.475^{a}(0.023)$ & $0.491^{\mathrm{a}}(0.023)$ & $0.511^{\mathrm{a}}(0.022)$ & $0.531^{\mathrm{a}}(0.024)$ \\
\hline \multicolumn{6}{|l|}{ Female } \\
\hline \multicolumn{6}{|l|}{ WC } \\
\hline Model 1 & $1.227^{\mathrm{a}}(0.068)$ & $1.001^{\mathrm{a}}(0.029)$ & $1.272^{\mathrm{a}}(0.029)$ & $1.332^{\mathrm{a}}(0.058)$ & $1.337^{\mathrm{a}}(0.063)$ \\
\hline Model 2 & $0.751^{\mathrm{a}}(0.044)$ & $0.763^{\mathrm{a}}(0.042)$ & $0.782^{\mathrm{a}}(0.043)$ & $0.791^{\mathrm{a}}(0.046)$ & $0.808^{\mathrm{a}}(0.047)$ \\
\hline Model 3 & $0.753^{\mathrm{a}}(0.032)$ & $0.786^{a}(0.032)$ & $0.788^{a}(0.032)$ & $0.801^{\mathrm{a}}(0.031)$ & $0.818^{\mathrm{a}}(0.035)$ \\
\hline \multicolumn{6}{|l|}{$\mathrm{WHtR}$} \\
\hline Model 1 & $0.749^{a}(0.029)$ & $0.747^{\mathrm{a}}(0.026)$ & $0.752^{a}(0.026)$ & $0.769^{\mathrm{a}}(0.029)$ & $0.802^{\mathrm{a}}(0.030)$ \\
\hline Model 2 & $0.361^{\mathrm{a}}(0.025)$ & $0.368(0.024)$ & $0.374^{\mathrm{a}}(0.024)$ & $0.379^{a}(0.024)$ & $0.390^{\mathrm{a}}(0.024)$ \\
\hline Model 3 & $0.356^{\mathrm{a}}(0.025)$ & $0.365^{\mathrm{a}}(0.025)$ & $0.362^{\mathrm{a}}(0.026)$ & $0.377^{\mathrm{a}}(0.026)$ & $0.390^{\mathrm{a}}(0.026)$ \\
\hline \multicolumn{6}{|l|}{ WHpR } \\
\hline Model 1 & $0.750^{\mathrm{a}}(0.032)$ & $0.701^{\mathrm{a}}(0.022)$ & $0.700^{\mathrm{a}}(0.027)$ & $0.707^{\mathrm{a}}(0.034)$ & $0.696^{\mathrm{a}}(0.048)$ \\
\hline Model 2 & $0.437^{\mathrm{a}}(0.026)$ & $0.442^{\mathrm{a}}(0.026)$ & $0.456^{\mathrm{a}}(0.026)$ & $0.463^{\mathrm{a}}(0.025)$ & $0.477^{\mathrm{a}}(0.026)$ \\
\hline Model 3 & $0.407^{\mathrm{a}}(0.023)$ & $0.421^{\mathrm{a}}(0.025)$ & $0.434^{\mathrm{a}}(0.023)$ & $0.444^{a}(0.022)$ & $0.450^{\mathrm{a}}(0.025)$ \\
\hline
\end{tabular}

Model 1 includes year only; Model 2 includes year, age, education, energy intake, physical activity, income, smoking and drinking history; Model 3 includes all the components of Model 2 and the urbanicity index

${ }^{\mathrm{a}} P<0.001$

Abbreviation: WC, waist circumference; WHtR, waist-to-height ratio; WHpR, waist-to-hip ratio

activity. Physical activity is an acknowledged protective factor for abdominal obesity because it is a major determinant of energy consumption [25, 33, 34] and is negatively associated with high $\mathrm{WC}, \mathrm{BMI}$ and $\mathrm{WHpR}$ [28]. In our study, we found that physical activity is especially meaningful in men and those with low values of abdominal obesity indicators. The divergent effect of drinking on the indicators between genders may also partly relate to education, income and employment. Highly educated women with better jobs and greater socializing skills may need to drink occasionally and are more likely to pay particular attention to their appearance [35]. In fact, studies on the association between alcohol consumption and abdominal obesity have presented inconsistent findings for either or both genders [36-39], which may be because alcohol is metabolized differently in men and women. However, the insight mechanism has not been well elucidated [35]. With respect to smoking, it generally had a significant negative association with the WC and WHtR in men. Divergent effects of smoking were also discussed in other literatures. One Chinese-population-based study demonstrated that regular smoking was associated with increased WC and WHtR after adjusting for BMI [40], while others claimed a negative association between smoking and body weight [41] or a positive association between smoking and increase in abdominal fat [34, 42]. The mechanisms through which smoking affects body weight and fat distribution are complex and still not fully understood. The effects of cigarette smoking on body weight are probably mediated by the smoking behavior which may serve as a behavioral alternative to eating, resulting in decreased food intake [41]. Besides, like many anti-obesity drugs, nicotine as a sympathomimetic agent, can reduce body weight by increasing energy expenditure [43]. 
Our study had several limitations. Detailed information regarding eating, drinking and smoking behaviors, as well as variables like occupation were not included in our database. Limitations also stem from the sample attrition. However, the rate of lost to follow-up was low relative to the large sample size and data was collected from fixed regions, so we assumed the missing data were uninformative. Sensitive analyses with imputed data were also conducted, providing results that were similar to those of our study. Despite these limitations, our study has several strengths. The longitudinal nature of variables at the individual, household and community levels was considered, and the effects on changes were analyzed in a comprehensive way. Moreover, the use of quantile regression allowed a more detailed and robust investigation of the distributions and trends of the indicators.

\section{Conclusions}

Over the 18-year study period, rapid increases in abdominal-obesity related measurements, WC, WHtR and WHpR were observed among Chinese adults. Specifically, these increases were greater at upper percentiles and greater in men. Age, physical activity, energy intake, drinking, smoking, educational level, income level and community-level urbanicity were associated with elevated abdominal obesity indicators, and the effects differed among percentiles and between genders. Regardless of which indicator is used, the remarkable increase of abdominal obesity in China is worthy of great attention. Furthermore, the increase is very likely to continue after 2011 accompanied with the ongoing processes of urbanicity and modernization. Therefore, effective strategies for preventing and controlling the epidemic of abdominal obesity are needed to diminish the negative effects on public health.

\section{Additional file}

Additional file 1: This file contains 4 figures and 3 tables that present additional results obtained in the study that we consider important to publish. Figure S1. depicts the shifts in distributions of WC, WHtR and WHpR for Chinese adults in each wave. Figure S2-S4. show the quantile curves by age of WC, WHtR and WHpR respectively for adults in each wave. Table S1-S3. provide the coefficients and standard errors from multivariate quantile regression for 10th, 25th, 50th, 75th and 90th percentiles of WC, WHtR and WHpR, respectively. (DOCX $11252 \mathrm{~kb}$ )

\section{Abbreviations}

BMI: Body mass index; CHNS: China Health and Nutrition Surveys; HC: Hip circumference; WC: Waist circumference; WHpR: Waist-to hip ratio; WHtR: Waist-to-height ratio

\section{Acknowledgements}

The authors thank the participants for their involvement in the survey. The authors also thank the National Institute for Nutrition and Health, Chinese Center for Disease Control and Prevention; the Carolina Population Center, University of North Carolina at Chapel Hill; The NIH and the Fogarty International Center, $\mathrm{NIH}$, for the collection and analysis files of the CHNS data from 1989 to 2011 and future surveys.

\section{Funding}

This research received no specific grant from any funding agency, commercial or not-for-profit sectors. The research uses data from China Health and Nutrition Survey (CHNS). The CHNS receives funding for data collection and analysis files from the National Institutes of Health $(\mathrm{NIH})$ (NIH grant P2C HD050924, T32 HD007168, R01DK104371, R01HL108427, R01 HD38700, P30 DK056350, R01 R01DK104371 and R01HL108427) and the NIH Fogarty grant (D43 TW009077).

\section{Availability of data and materials}

The datasets analyzed in the current study are not publicly available. Request to access datasets can be made to the corresponding author.

\section{Authors' contributions}

The authors' responsibilities were as follows: XQ, CS, HW and ZW designed the overall study and wrote the manuscript; $X Q, G Q$ and $Z W$ analyzed and the data; CS, BZ, GQ and HW interpreted the data and reviewed the manuscript; all authors read and approved the final version of the paper.

Ethics approval and consent to participate

Not applicable.

\section{Consent for publication}

Not applicable.

\section{Competing interests}

The authors declare that they have no competing interests.

\section{Publisher's Note}

Springer Nature remains neutral with regard to jurisdictional claims in published maps and institutional affiliations.

Received: 11 October 2018 Accepted: 30 April 2019

Published online: 06 June 2019

\section{References}

1. Bener A, Yousafzai MT, Darwish S, Al-Hamaq AO, Nasralla EA, Abdul-Ghani M. Obesity index that better predict metabolic syndrome: body mass index, waist circumference, waist hip ratio, or waist height ratio. J Obes. 2013;2013: 269038

2. Park JH, Cho IC, Kim YS, Kim SK, Min SK, Kye SS. Body mass index, waist-tohip ratio, and metabolic syndrome as predictors of middle-aged men's health. Korean J Urol. 2015;56(5):386-92.

3. Niu J, Seo DC. Central obesity and hypertension in Chinese adults: a 12-year longitudinal examination. Prev Med. 2014;62:113-8.

4. Tanamas SK, Shaw JE, Backholer K, Magliano DJ, Peeters A. Twelve-year weight change, waist circumference change and incident obesity: the Australian diabetes, obesity and lifestyle study. Obesity (Silver Spring). 2014; 22(6):1538-45

5. Han TS, Correa E, Lean MEJ, Lee DM, O Neill TW, Bartfai G, Forti G, Giwercman A, Kula K, Pendleton N, et al. Changes in prevalence of obesity and high waist circumference over four years across European regions: the European male ageing study (EMAS). Endocrine. 2017;55(2):456-69.

6. Ford ES, Maynard LM, Li C. Trends in mean waist circumference and abdominal obesity among US adults, 1999-2012. JAMA. 2014;312(11):1151-3.

7. P D, Wang HJ, Zhang B, Qi SF, Mi YJ, Liu DW, Tian QB. Prevalence of abdominal obesity among Chinese adults in 2011. J Epidemiol. 2017; 27(6):282-6

8. Zhang M, Zhao $Y$, Wang G, Zhang $H$, Ren $Y$, Wang B, Zhang L, Yang $X$, Han C, Pang $C$, et al. Body mass index and waist circumference combined predicts obesity-related hypertension better than either alone in a rural Chinese population. Sci Rep. 2016;6(1):31935.

9. Tanamas SK, Ng WL, Backholer K, Hodge A, Zimmet PZ, Peeters A. Quantifying the proportion of deaths due to body mass index- and waist circumference-defined obesity. Obesity (Silver Spring). 2016;24(3):735-42.

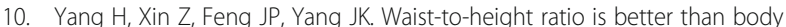
mass index and waist circumference as a screening criterion for metabolic syndrome in Han Chinese adults. Medicine. 2017;96(39):e8192.

11. Ashwell M, Gunn P, Gibson S. Waist-to-height ratio is a better screening too than waist circumference and BMI for adult cardiometabolic risk factors: systematic review and meta-analysis. Obes Rev. 2012;13(3):275-86. 
12. Shen S, Lu Y, Qi H, Li F, Shen Z, Wu L, Yang C, Wang L, Shui K, Yao W, et al. Waist-to-height ratio is an effective indicator for comprehensive cardiovascular health. Sci Rep. 2017;7:43046.

13. Cameron AJ, Magliano DJ, Shaw JE, Zimmet PZ, Carstensen B, Alberti KG, Tuomilehto J, Barr EL, Pauvaday VK, Kowlessur S, et al. The influence of hip circumference on the relationship between abdominal obesity and mortality. Int J Epidemiol. 2012;41(2):484-94.

14. Cameron AJ, Magliano DJ, Söderberg S. A systematic review of the impact of including both waist and hip circumference in risk models for cardiovascular diseases, diabetes and mortality. Obes Rev. 2013;14(1):86-94.

15. de Koning L, Merchant AT, Pogue J, Anand SS. Waist circumference and waist-to-hip ratio as predictors of cardiovascular events: meta-regression analysis of prospective studies. Eur Heart J. 2007;28(7):850-6.

16. Pei L, Cheng Y, Kang Y, Yuan S, Yan H. Association of obesity with socioeconomic status among adults of ages 18 to 80 years in rural Northwest China. BMC Public Health. 2015;15:160.

17. Cohen AK, Rai M, Rehkopf DH, Abrams B. Educational attainment and obesity: a systematic review. Obes Rev. 2013;14(12):989-1005.

18. Bottai M, Frongillo EA, Sui X, O'Neill JR, McKeown RE, Burns TL, Liese AD, Blair SN, Pate RR. Use of quantile regression to investigate the longitudinal association between physical activity and body mass index. Obesity (Silver Spring). 2014;22(5):E149-56

19. Popkin BM, S D, Zhai F, Zhang B: cohort profile: the China health and nutrition survey--monitoring and understanding socio-economic and health change in China, 1989-2011. Int J Epidemiol 2010, 39(6):1435-1440.

20. Zhang B, Zhai FY, SF D, Popkin BM. The China health and nutrition survey, 1989-2011. Obes Rev. 2014;15(Suppl 1):2-7.

21. Du S, Mroz TA, Zhai F, Popkin BM. Rapid income growth adversely affects diet quality in China--particularly for the poor! Soc Sci Med. 2004;59(7): 1505-15.

22. Jones-Smith JC, Popkin BM. Understanding community context and adult health changes in China: development of an urbanicity scale. Soc Sci Med. 2010;71(8):1436-46.

23. T D SX, Yin P, Huo R, Ni C, Yu X. Increasing trends in central obesity among Chinese adults with normal body mass index, 1993-2009. BMC Public Health. 2013;13:327.

24. Xi B, Liang Y, He T, Reilly KH, Hu Y, Wang Q, Yan Y, Mi J. Secular trends in the prevalence of general and abdominal obesity among Chinese adults, 1993-2009. Obes Rev. 2012;13(3):287-96.

25. Ladabaum U, Mannalithara A, Myer PA, Singh G. Obesity, abdominal obesity, physical activity, and caloric intake in US adults: 1988 to 2010. Am J Med. 2014;127(8):717-27 e712.

26. Lahtikoski M, Pietinen P, Nnist SM, Vartiainen E. Trends in waist-to-hip ratio and its determinants in adults in Finland from 1987 to 1997. Am J Clin Nutr. 2000;72(6): :1436-44.

27. Comitato R, Saba A, Turrini A, Arganini C, Virgili F. Sex hormones and macronutrient metabolism. Crit Rev Food Sci Nutr. 2015;55(2):227-41.

28. Czernichow S, Bertrais S, Preziosi P, Galan P, Hercberg S, Oppert JM. Indicators of abdominal adiposity in middle-aged participants of the SU.VI. MAX study: relationships with educational level, smoking status and physical inactivity. Diabetes Metab. 2004;30(2):153-9.

29. Zhao PP, Gu XL, Qian DF, Yang F. Socioeconomic disparities in abdominal obesity over the life course in China. Int J Equity Health. 2018;17.

30. Pradeepa R, Anjana RM, Joshi SR, Bhansali A, Deepa M, Joshi PP, Dhandania VK, Madhu V, Rao PV, Geetha L, et al. Prevalence of generalized \& abdominal obesity in urban \& rural India- the ICMR-INDIAB study (phase-I) [ICMR-INDIAB-3]. Indian J Med Res. 2015;142:139-50.

31. Janghorbani M, Amini M, Rezvanian H, Gouya MM, Delavari A, Alikhani S, Mahdavi A. Association of body mass index and abdominal obesity with marital status in adults. Archives of Iranian medicine. 2008;11(3):274-81.

32. Zhai $Y$, Fang HY, Yu WT, Yu DM, Zhao LY, Liang XF, Zhao WH. Changes in waist circumference and abdominal obesity among Chinese adults over a ten-year period. Biomed Environ Sci. 2017;30(5):315-22.

33. López-Sobaler AM, Rodríguez-Rodríguez E, Aranceta-Bartrina J, Gil Á, González-Gross M, Serra-Majem L, Varela-Moreiras G, Ortega RM. General and abdominal obesity is related to physical activity, smoking and sleeping Behaviours and mediated by the educational level: findings from the ANIBES study in Spain. PLoS One. 2016;11(12):e0169027.

34. Koh-Banerjee P, Chu NF, Spiegelman D, Rosner B, Colditz G, Willett W, Rimm E. Prospective study of the association of changes in dietary intake, physical activity, alcohol consumption, and smoking with 9-y gain in waist circumference among 16587 US men. Am J Clin Nutr. 2003;78(4):719-27.

35. Buemann B, Astrup A. How does the body deal with energy from alcohol? Nutrition. 2001:17(7-8):638-41.

36. Sakurai Y, Umeda T, Shinchi K, Honjo S, Wakabayashi K, Todoroki I, Nishikawa H, Ogawa S, Katsurada M. Relation of total and beverage-specific alcohol intake to body mass index and waist-to-hip ratio: a study of selfdefense officials in Japan. Eur J Epidemiol. 1997;13(8):893-8.

37. Da Rocha TF, Hasselmann MH, Chaves Curioni C, Bezerra FF, Faerstein E. Alcohol consumption is associated with DXA measurement of adiposity: the Pró-Saúde study, Brazil. Eur J Nutr. 2017;56(5):1983-91.

38. Funtikova AN, Subirana I, Gomez SF, Fitó M, Elosua R, Benítez-Arciniega AA, Schröder H. Soft drink consumption is positively associated with increased waist circumference and 10-year incidence of abdominal obesity in Spanish Adults1-3. J Nutr. 2015:145(2):328-34.

39. Park K-Y, Park H-K, Hwang H-s: relationship between abdominal obesity and alcohol drinking pattern in normal-weight, middle-aged adults: the Korea National Health and nutrition examination survey 2008-2013. Public Health Nutr 2017, 20(12):2192-2200

40. Lv J, Chen W, Sun D, Li SX, Millwood IY, Smith M, Guo Y, Bian Z, Yu CQ, Zhou HY, et al. Gender-specific association between tobacco smoking and central obesity among 0.5 million Chinese people: the China Kadoorie biobank study. PLoS One. 2015;10(4).

41. Audrain-McGovern J, Benowitz NL. Cigarette smoking, nicotine, and body weight. Clin Pharmacol Ther. 2011;90(1):164-8.

42. Barrett-Connor E, Khaw KT. Cigarette smoking and increased central adiposity. Ann Intern Med. 1989;111(10):783-7.

43. Andersson K, Arner P. Systemic nicotine stimulates human adipose tissue lipolysis through local cholinergic and catecholaminergic receptors. Int $J$ Obes Relat Metab Disord. 2001;25(8):1225-32.

\section{Ready to submit your research? Choose BMC and benefit from:}

- fast, convenient online submission

- thorough peer review by experienced researchers in your field

- rapid publication on acceptance

- support for research data, including large and complex data types

- gold Open Access which fosters wider collaboration and increased citations

- maximum visibility for your research: over $100 \mathrm{M}$ website views per year

At BMC, research is always in progress.

Learn more biomedcentral.com/submissions 